\title{
Effects of Transaction Costs and Discount Rate on the Banking Decision of Emission Permits Trading*
}

\author{
Karima Fredj $^{1, *}$, Alain Jean-Marie ${ }^{2}$, Guiomar Mart'́n-Herr'á $^{3}$, Mabel Tidball ${ }^{4}$ \\ ${ }^{1}$ Department of Economics, University of Northern British Columbia, Canada \\ ${ }^{2}$ INRIA and UMR LIRMM, 161 Rue Ada, 34095 Montpellier Cedex 5, France \\ ${ }^{3}$ IMUVA, Universidad de Valladolid, Avda. Valle Esgueva, 6, 47011-Valladolid, Spain \\ ${ }^{4}$ INRA, UMR 5474 Lameta, F-34000 Montpellier, France
}

Received May 29, 2019; Revised October 14, 2019; Accepted October 17, 2019

Copyright (C) 2020 by authors, all rights reserved. Authors agree that this article remains permanently open access under the terms of the Creative Commons Attribution License 4.0 International License

\begin{abstract}
This paper characterizes and compares the optimal and the strategic behaviour of two countries or firms that minimize costs facing emission standards. Emission standards can be reached through emission reduction, banking or borrowing, and emission trading in a given and fixed planning $\mathrm{h}$ orizon. Our model extends the existing theoretical models in this area of research in two directions mainly. First, we revisit the model proposed by Rubin (1996) to introduce and study the impacts of transaction costs in treadable emission markets. Second, we extend Stavins' (1995) work from a static to a dynamic setting. We analyze the case with and without transaction costs and the case with and without discount rate. We characterize solutions and equilibria in each case and, depending on the initial allocation, characterize the buyer and seller in the emission trading market. Our main findings extend Rubin's paper proving that agents' equilibrium is not efficient when transaction cost are positive and expand Stavins' results to a dynamic framework.
\end{abstract}

Keywords Emission Permits Trading, Transaction Costs, Intertemporal Continuous Time Framework

\section{Introduction}

Emission permits trading is one of the economic instruments that helps countries (and firms) to comply and achieve their environmental targets as determined by international treaties (and by regulatory agencies) in a cost-effective way (e.g. Cropper and Oates, 1992; Tietenberg, 1985; Montgomery, 1972; Cronshaw and Kruse, 1996). Under this system, each agent (firm or country) tries to reach its emission standard through abatement or by buying additional pollution permits from other (overcomplying) agents. An overcomplying firm or country usually has emission rates that are below its preassigned target and can therefore chose to sell the extra permits, bank them for future use, or do both (e.g. Dales, 1968; Croker, 1996).

Rubin (1996) provides a general study of emission trading, banking, and borrowing that extends existing literature by using an intertemporal continuous-time framework within an optimal control model. He provides equilibrium time paths for emissions as well as qualitative impacts of emissions' borrowing on permit prices, emission stream and total social damages. His results confirm that decentralized firms' behavior leads to the least-cost solution that can be reached under joint-cost minimization. Rubin's (1996) work, however, does not take into account transaction costs related to permits' trading, which represents a significant limitation given the importance and impacts of transaction costs in tradeable markets that have been discussed and proven by previous studies (e.g. Baumol and Oates, 1988; Hahn and Hester, 1989; Tripp and Dudek, 1989; Stavins, 1995).

Stavins (1995) points out to the importance of transaction costs and its significant impact on the tradeable permits markets, in terms of increased abatement costs and reduced trading levels in a static framework. He argues that transaction costs affect

\footnotetext{
*The third author has been partially supported by MEC under project ECO2017-82227-P (AEI) and by Junta de Castilla y León VA024P17 and VA105G18 co-financed by FEDER funds (EU). The second, third and fourth authors acknowledge the support by COST Action IS1104.
} 
negatively the relative cost-effectiveness of permits trading, otherwise exaggerated, and make them more sensitive to initial permits allocation. Stavin's findings however were limited to a static framework and do not offer results that apply to more general dynamic intertemporal framework.

To address the above limitations, we extend the existing theoretical models in this area in two main directions. We revisit Rubin's (1996) to introduce and study the impacts of transaction costs in tradeable emission markets. At the same time we extend Stavins' (1995) work to offer a more comprehensive study of intertemporal emission treading within continuous time framework. This allows us to determine and compare equilibrium time paths for emissions, emissions banking and borrowing, and permit prices as well as the cost-effectiveness and efficiency of emission permits trading under different scenarios that distinguish the cases of positive and zero discount rate, with and without transaction cost. It also allows us to determine how initial allocations of standards can determine the direction of trade (buyer and seller) between firms or countries in the emission trading market.

\section{The Model}

We consider two agents (firms or countries) $(i=1,2)$, Each of whom faces an emission standard $S_{i}(t)$ to be reached by the end of period $t$. If a firm or country $i$ pollutes above its allocated standard $S_{i}(t)$, it has the option to buy extra permits in order to meet its target. On the other hand, if a firm (country) pollutes less than what it is allowed by the allocated target, then it has three possible options: It can either sell the excess of its pollution permits, it can bank them for future use, or it can do both and find an optimal combination where some of the excess permits are banked and the rest sold to other firm (country). We denote by $B_{i}(t)$ the level of emissions that are banked at time $t$.

Each firm (or country) controls the quantity of emissions that it produces $\left(e_{i}(t)>0\right)$ and the quantity of emissions' permits $y_{i}(t)$ that it buys $\left(y_{i}(t)>0\right)$ or sells $\left(y_{i}(t)<0\right)$. The price of permits , $p(t)$, is determined in the market by the equilibrium (market clearing) conditions over the planning horizon $[0, T]$.

Following the literature (e.g. Rubin 1996; Montgomery 1972), we consider an abatement cost function $C_{i}\left(e_{i}(t)\right)$ that is strictly decreasing and convex in the emission rates $\left(C_{i}^{\prime}\left(e_{i}(t)\right)<0\right.$, and $C_{i}^{\prime \prime}\left(e_{i}(t)>0\right)$. We also assume that emissions standard are strictly decreasing in time $\left(S_{i}^{\prime}(t)<0\right)$. This assumption is easily justified by tighter environmental regulations resulting from higher environmental degradation (e.g. Rubin 1996).

Let $\tau_{i}(y)$ be the transaction cost related to emission trading. We assume that the transaction cost function is convex in the quantity of emission permits $\left(\tau_{i}^{\prime \prime}(y(t))>0\right)$ and symmetric $\left(\tau_{i}(y(t))=\tau_{i}(-y(t))\right)$. We also assume that transaction costs are shared by the selling and buying firms (or countries).

Our main objectives are to study and compare the joint-cost problem and the agents' problem in terms of (1) emissions' rates, banking and borrowing strategies, and (2) cost effectiveness under few different scenarios: with transaction cost $\left(\tau_{i}(y(t))>0\right)$ and without transaction cost $\left(\tau_{i}(y(t))=0\right)$ considering both the case of a positive discount $(r>0)$ rate and zero discount rate $(r=0)$. This will allow us to contrast the results we obtain in a more generalized and dynamic framework to the existing findings.

\section{The joint-cost minimization problem}

The joint-cost minimization problem represents the situation where a social planner (respectively international regulator) allocates the total emission standards between two firms (respectively countries) and aims at minimizing their joint abatement costs. We will first study the joint-cost problem in the general case of a positive discount rate, then look at the particular case with no discounting.

\subsection{General case : positive discount rate}

Let $B(t)$ be the aggregate banked emissions, and $S(t)$ the aggregate standards of emissions. The joint-cost problem $(\mathcal{J C P})$ in this case can be represented by the following objective function and constraints: ${ }^{1}$

$$
\begin{gathered}
J^{* *}=\min _{\left\{e_{1}(t), e_{2}(t)\right\}} \int_{0}^{T} e^{-r t}\left[C_{1}\left(e_{1}(t)\right)+C_{2}\left(e_{2}(t)\right)\right] d t \\
\text { s.t. } \dot{B}(t)=S(t)-e_{1}(t)-e_{2}(t), \quad B(0)=0, \quad B(t)=B_{1}(t)+B_{2}(t) \geq 0, \quad e_{i}(t) \geq 0 .
\end{gathered}
$$

In equation (1) $r$ denotes the discount rate. The constraints expressed in equations (2) represent, in the same order, the dynamics of banked emissions permits stating that the quantity of additional emissions that can be banked at each point of time $t$ is equal to the aggregated emission permits allocated as standard to both firms (countries) reduced by the emissions they jointly produce; the

\footnotetext{
${ }^{1}$ We will use the superscript $(* *)$ to indicate the optimal solutions for the joint-cost minimization problem.
} 
conditions on initial stock of banked emissions to be equal to zero; the conditions on the aggregate stock of banked emissions; and the emission rates $e_{i}(t)$, to be non-negative at each point of time $t$.

The Lagrangian associated to this problem can be written as: ${ }^{2}$

$$
L=C_{1}\left(e_{1}\right)+C_{2}\left(e_{2}\right)+\Lambda\left(S_{1}+S_{2}-e_{1}-e_{2}\right)-\Phi B
$$

where $\Lambda$ is the costate variable associated with the dynamic equation of banked emissions, and $\Phi$ the Lagrangian multiplier function associated with the non-negativity constraint of the total banked emissions.

The first-order conditions are:

$$
\begin{aligned}
C_{i}^{\prime}\left(e_{i}\right) & =\Lambda, i=1,2 \\
\dot{\Lambda} & =r \Lambda+\Phi, \quad B \geq 0, \quad \Phi \geq 0, \quad B \Phi=0 ; \\
\dot{B} & =S_{1}+S_{2}-e_{1}-e_{2}, \quad \Lambda(T) \leq 0, \quad B(T) \Lambda(T)=0 .
\end{aligned}
$$

From (equation 3), we can deduce that the minimizing emission rates are the same for both firms (countries). They correspond to equal marginal abatement costs of emissions and marginal cost of banking, the latter being the same for both firms. Equation (4) describes the dynamics of the marginal value of an additional unit of emission banked, and equation (5) reproduces the state equation constraint that has to be satisfied for the whole optimization period.

The above necessary conditions are similar to those found in the joint optimization problem with no transaction costs (Rubin 1996) given that the transaction costs are neutralized in the case of joint maximization problem. by

let $\Lambda_{0}$ be the initial value of the costate variable, the optimal solutions to the $\mathcal{J C P}$ with and without banking are characterized

Theorem 1 In the joint-cost problem with positive discount rate,

- When firms (countries) choose to bank a positive amount of emission permits (as long as $B(t)>0)$, the optimal emissions rate $e_{i}\left(t, \Lambda_{0}\right)$ are solutions of

$$
C_{1}^{\prime}\left(e_{1}\right)=C_{2}^{\prime}\left(e_{2}\right)=\Lambda_{0} e^{r t}
$$

and the banked emissions $B(t)$ and $\Lambda_{0}$ solutions of

$$
\dot{B}=S_{1}+S_{2}-e_{1}-e_{2}, \quad B(0)=0, \text { and } B(T)=0
$$

- When firms (countries) choose not to bank emission permits, the optimal emission rates $e_{i}(t)$ are solutions of the system equations

$$
C_{1}^{\prime}\left(e_{1}\right)=C_{2}^{\prime}\left(e_{2}\right) \quad \text { and } \quad S_{1}+S_{2}-e_{1}-e_{2}=0 .
$$

This solution is valid as long as $\Phi=C_{1}^{\prime \prime}\left(e_{1}\right) \dot{e_{1}}-r C_{1}^{\prime}\left(e_{1}\right) \geq 0$.

Remark $1 \dot{B}(0)>0$ (respectively $\dot{B}(0)<0$ ) implies that banking is possible (respectively not possible) at the beginning of the time interval. In the same way $\Phi(0)=C_{i}^{\prime \prime}\left(e_{i}(0)\right) e_{i}(0)-r C_{i}^{\prime}\left(e_{i}(0)\right) \geq 0\left(\right.$ respectively $\Phi(0)=C_{i}^{\prime \prime}\left(e_{i}(0)\right) e_{i}(0)-r C_{i}^{\prime}\left(e_{i}(0)\right) \leq$ $0)$ implies that no-banking is possible (respectively not possible) at the beginning of the time interval.

Next, we present an illustrative example of the case where the optimal emission rates are decreasing over time and where the emission banking rates are always positive over the whole optimization period. In this situation both banking and no banking occur along the optimal trajectory.

Example 2 Let us consider: $T=1, S_{1}(t)=1-t, S_{2}(t)=2-2 t, r=2, C_{1}=C_{2}=C, C(e)=-a e+(b / 2) e^{2}$. The cost functions being the same, optimal emissions $e_{i}$ will also be the same. With the numerical values $a=1.4, b=1$, we obtain the following optimal solution $e^{* *}=e_{i}^{* *}, B^{* *}$, are as illustrated in Figure 1.

The above characterizes the general optimal solution considering a positive discount rate $(r>0)$. Next, we study the particular case of a null discount rate for which we obtain additional results about the banking strategies and conditions.

\footnotetext{
${ }^{2}$ The time argument is eliminated when no confusion can arise. In the Lagrangian equation we do not consider the positivity of $e_{i}$. We do, however, check it a posteriori, which should not be an issue in this case. Indeed, because the cost functions are decreasing, an interior solution, if it exists, should be superior than a corner solution.
} 

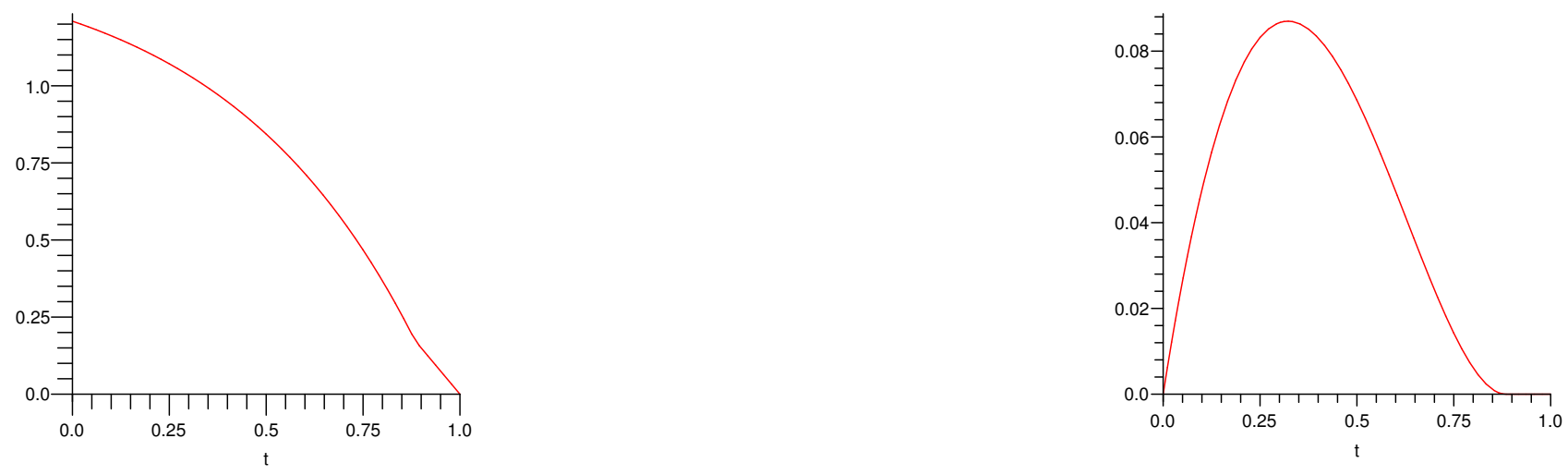

Figure 1. $e^{* *}(t)$ (left); $B^{* *}(t)$ (right)

\subsection{Particular case : zero discount rate}

The assumption of a zero discount rate is reasonable given the short time horizon we are dealing with in this problem. In addition, it is appealing from an environmentalist point of view and given the inter-generational equity perspective it involves.

The necessary conditions yielding from this particular case are mainly the same equations (3) to (5) found for the general case with positive discount rate. The main difference is captured by condition (4). The latter indicates that when firms or countries, globally, bank a positive quantity of emission permits, the marginal value of an additional unit of emissions banked remains constant in the case of zero discount rate in contrast to the case of positive discount rate. The following theorem summarizes the solutions implied by these conditions:

Theorem 3 The optimal interior solutions for the emission rates $\left(e_{i}^{* *}>0\right)$ in the $\mathcal{J C P}$ when the discount rate is zero, has the following properties:

$$
B^{* *}(t)>0, \forall t \in(0, T) ; \quad B(0)=B(T)=0,
$$

and $e_{i}^{* *}$ are constant, solutions of

$$
\frac{\bar{S}_{1}+\bar{S}_{2}}{T}=e_{1}+e_{2} \quad \text { and } \quad C_{1}^{\prime}\left(e_{1}\right)=C_{2}^{\prime}\left(e_{2}\right)
$$

where $\bar{S}_{i}=\int_{0}^{T} S_{i}(t) d t$ is the cumulative emission standards over the planning horizon $[0, T]$.

Proof. Note that the case of $\Phi$ strictly positive in some open interval of time is not possible. For in this case $B$ would be identically equal to zero, and from (2) we would have $S_{1}+S_{2}=e_{1}+e_{2}$. Given that $S_{i}$ is a decreasing function of time, we have, on one hand, $\dot{S}_{1}+\dot{S}_{2}=\dot{e_{1}}+\dot{e_{2}}<0$; meaning that $\dot{e}_{i}<0$ for at least one of the agents (firm or country) $i=1,2$. For that agent $i$ we have, from equation $C_{i}^{\prime}\left(e_{i}\right)=\Lambda$ and differentiating with respect to time, $\dot{\Lambda}=C_{i}^{\prime \prime}\left(e_{i}\right) \dot{e}_{i}<0$ ( $C_{i}$ being convex). On the other hand, we have $\dot{\Lambda}=\Phi$ that is positive (Equation (4)), which leads to a contradiction. Hence, $\Phi$ can only be identically equal to zero. Referring again to first-order condition (4), $\Lambda$ is a constant and $B(t) \geq 0$.

Moreover $C_{1}^{\prime}\left(e_{1}\right)=C_{2}^{\prime}\left(e_{2}\right)=\Lambda$, implies that both $e_{i}$ are constant over time.

$B$ is the solution of $\dot{B}=S_{1}+S_{2}-e_{1}-e_{2}, \quad B(0)=0, \quad B(T)=0$. With $e_{i}$ constant, $B(T)=0$ implies $\frac{\overline{S_{1}}+\overline{S_{2}}}{T}=e_{1}+e_{2}$. This completes the proof.

\subsection{The effect of discount rate}

The impact of interest rate on the optimal banking option and emissions rate are summarized in

Theorem 4 For the joint-cost minimization problem, "no banking" is never an optimal solution when the discount rate is zero. This is not necessarily the case when the discount rate is strictly positive. Moreover, emissions are constant in the zero-discount case, and strictly decreasing in the case of positive discount rate.

Proof. In Theorem 3 we proved that no banking is never a solution when discount rate is zero. Note that this is essentially due to the fact that $S_{i}$ are decreasing functions of time. Example 2 shows that it cannot be the case with positive discount rate.

The second statement is an obvious corollary of Theorem 1 and Theorem 3. 


\section{The optimal control problem}

In this section we focus on the scenario where each agent $i$ (firm or country) tries to minimize individually its total costs while facing emission standard constraints $\left(S_{i}(t)\right)$. The total costs are incurred from emissions' abatement and permits' trading. Each firm (country) chooses the optimal rate of emissions to abate and quantity of permits to trade in order to minimize its total incurred cost while respecting the constraints of banking dynamics.

By analogy to the $\mathcal{J C P}$, we study the general case with positive discount rate as well as the particular case of a zero discount rate. We further contrast two sub-cases: the first one considers positive transaction costs, similar to Stavins (1995) however in a dynamic setting, and the second one involves no transaction costs in permits trading as in Rubin (1996). This section concludes with a comparison of all the different scenarios' outcomes.

\subsection{Positive discount rate without transaction cost}

Under this scenario, we look into each agent's optimization problem $\mathcal{A P}$, when the discount rate is positive and no transaction costs are involved (Rubin's case). The problem for each firm (country) $i$ can be written as: ${ }^{3}$

$$
\begin{aligned}
J_{i}^{*} & :=\min _{e_{i}(t), y_{i}(t)} \int_{0}^{T} e^{-r t}\left[C_{i}\left(e_{i}(t)\right)+p(t) y_{i}(t)\right] d t \\
& \text { s.t.: } \dot{B}_{i}(t)=S_{i}(t)-e_{i}(t)+y_{i}(t), \quad B_{i}(0)=0, \quad B_{i}(t) \geq 0, \quad e_{i}(t) \geq 0 .
\end{aligned}
$$

Additional constraints are necessary to fully specify the problem: The permit unit price $p(t)$ is determined by the equilibrium condition and the terminal stock condition, respectively:

$$
\begin{gathered}
y_{1}^{*}+y_{2}^{*}=0, \\
p^{*}(T)\left(B_{1}^{*}(T)+B_{2}^{*}(T)\right)=0 .
\end{gathered}
$$

Equation (6) establishes the dynamics of the emission permits banking in the $\mathcal{A P}$. Compared to the $\mathcal{J C P}$, this equation includes the amount of permits traded by firms (countries). Equation (7) guarantees the clearing of tradeable permits' market, simply requiring that the quantity of permits sold by one firm (country) are bought by the other one. Equation (8) states that, at the terminal period, either the unit price of emissions in the bank or the total stock of permits are null.

Following Rubin (1996) we also consider that quantities of permits traded $\left(y_{i}\right)$ are bounded, i.e. $-A_{i}(t) \leq y_{i}(t) \leq D_{i}(t)$, and that firms (countries) do not buy or sell permits at the maximal or minimal rates.

The problem can, then, be solved using optimal control theory for each firm (country). The Lagrangian associated to each agent $i$ writes as:

$$
L_{i}=C_{i}\left(e_{i}\right)+p(t) y_{i}+\lambda_{i}\left(S_{i}-e_{i}+y_{i}\right)-\phi_{i} B_{i},
$$

$\lambda_{i}$ represents the costae variable associated with the dynamic equation of banked emissions for agent $i$, and $\phi_{i}$ the Lagrangian multiplier function associated with the non-negativity constraint of its total banked emission until time $t$. The necessary optimal and market clearing conditions are given by:

$$
\begin{aligned}
& C_{i}^{\prime}\left(e_{i}\right)=\lambda_{i}=-p, \quad \dot{\lambda}_{i}=r \lambda_{i}+\phi_{i}, \quad \dot{B}_{i}=S_{i}-e_{i}+y_{i}, \quad B_{i}(0)=0 \\
& y_{1}=-y_{2}, \quad \phi_{i} \geq 0, \quad B_{i} \geq 0, \quad \phi_{i} B_{i}=0, \quad \lambda_{i}(T) \leq 0, \quad B_{i}(T)=0 .
\end{aligned}
$$

Knowing that $\dot{B}=\dot{B}_{1}+\dot{B}_{2}$ and $B(0)=B(T)=0$, we can easily prove the following result that completes Rubin's:

Theorem 5 When the discount rate is positive and transaction costs are null, the equilibrium solutions satisfy: $-p=\Lambda$, $e_{i}^{*}=e_{i}^{* *}$ and $\phi_{i}=\phi_{j}=\Phi$. Moreover, at the equilibrium, the quantities of permits traded $y_{i}(t)$ are as follows:

- If both firms (countries) bank, then $y_{1}(t)$ satisfies $\dot{B}_{1}=S_{1}-e_{1}+y_{1}, B_{1}(0)=0=B_{1}(T)$ and $B_{2}=B^{*}-B_{1}$.

- If no firm (country) banks, then $y_{1}(t)$ satisfies $S_{1}=e_{1}-y_{1}$, or equivalently $S_{2}=e_{2}+y_{1}$.

Remark 2 The existence of a solution in the case with banking is guaranteed by the equilibrium condition $\dot{B}=S_{1}+S_{2}-e_{1}-e_{2}$. In the case of no banking this condition becomes $S_{1}+S_{2}=e_{1}+e_{2}$.

As a corollary of Theorems 1 and 5, we find the Rubin's main result:

Theorem $6 \mathcal{A P}$ is efficient.

\footnotetext{
${ }^{3}$ We will use the superscript $(*)$ to indicate the optimal solutions for the firm's minimization problem.
} 
In the particular case where the firms have symmetric cost functions, we can solve for the exact quantity of permits traded:

Theorem 7 When cost functions are symmetric, $y_{1}=\frac{S_{2}-S_{1}}{2}$ is the exact solution for the permits quantity that is traded between the two firms. The buyer would be the player with a higher emissions standard.

Proof. If $C_{1}=C_{2}=C$, then $e_{1}=e_{2}=e$. Considering $y_{1}=\frac{S_{2}-S_{1}}{2}$, then $\dot{B}_{1}=\dot{B}_{2}=\frac{\dot{B}}{2}$, which means that $\dot{B}_{i}$ is positive or zero when $\dot{B}$ is positive or zero.

\subsection{Particular case: zero discount rate.}

Under this particular scenario, the emphasis is more on the transaction costs and their importance in determining the optimal choices by firms (countries). The $\mathcal{A P}$ is:

$$
\begin{aligned}
J_{i}^{*}:=\quad \min _{e_{i}, y_{i}} & \int_{0}^{T}\left[C_{i}\left(e_{i}(t)\right)+p(t) y_{i}(t)+\tau\left(y_{i}(t)\right)\right] d t \\
& \text { s.t. } \dot{B}_{i}=S_{i}-e_{i}+y_{i}, \quad B_{i}(0)=0, \quad B_{i}(t) \geq 0, \quad e_{i}(t) \geq 0 . \\
& y_{1}^{*}+y_{2}^{*}=0, \\
& p^{*}(T)\left(B_{1}^{*}(T)+B_{2}^{*}(T)\right)=0 .
\end{aligned}
$$

The Lagrangian associated to this problem is:

$$
L_{i}=C_{i}\left(e_{i}\right)+p(t) y_{i}+\tau\left(y_{i}\right)+\lambda_{i}\left(S_{i}-e_{i}+y_{i}\right)-\phi_{i} B_{i},
$$

and the first-order conditions are:

$$
\begin{aligned}
& \frac{\partial L_{i}}{\partial e_{i}}=0 \quad \Longleftrightarrow C_{i}^{\prime}\left(e_{i}\right)=\lambda_{i}, \\
& \frac{\partial L_{i}}{\partial y_{i}}=0 \quad \Longleftrightarrow p+\lambda_{i}+\tau^{\prime}\left(y_{i}\right)=0, \\
& \dot{\lambda}_{i}=\phi_{i}, \quad \phi_{i} \geq 0, \quad B_{i} \geq 0, \quad \phi_{i} B_{i}=0, \\
& \lambda_{i}(T) \leq 0, \quad B_{i}(T) \lambda_{i}(T)=0 .
\end{aligned}
$$

According to Equation (12), the marginal abatement cost is equal to the marginal value of one additional unit of emissions permit in the firm's (country's) bank, which are negative given the properties of the abatement cost function. Equation (13) requires that at the equilibrium, the unit price of permits $(p)$ is equal to the sum of its marginal cost of banking and the marginal transaction cost. Equation (14) implies that the marginal value of an additional unit of emission banked is increasing (becomes less negative) when the non-negativity constraint is binding, and is constant otherwise. Finally, Equation (15) reflects the final terminal conditions on the firm's (country's) total banked permits and its costae variable. Furthermore, having a negative incremental value for the additional unit of emissions in a firm's (country's) bank reflects the fact that the abatement cost as well as the value of total cost will be lower when the firm (country) has additional unit of emissions in its bank (see Rubin 1996).

In the next two theorems, we characterize the equilibrium solutions for the firm's (country's) problem with and without transactions cost. Theorem 7 proves that independently of the existence or not of transaction costs "no banking" $\left(B_{i}\right.$ identically zero) is never a solution for the firm's cost minimization problem. This was the case for the $\mathcal{J C P}$.

Theorem 8 The case $\phi_{i}>0$ (that is, $B_{i}$ identically zero) cannot be an equilibrium for firm (country) $i$ in any interval of time.

Proof. We consider two possibilities: either only one of the firms (countries) or both have $\phi_{i}>0$.

- Let $\phi_{i}>0$ and $e_{i}>0$ for $i=1,2$. Then $B_{i} \equiv 0$ implies that $S_{1}+S_{2}=e_{1}+e_{2}$. We can then apply similar argument as in Theorem 3. If $e_{i}=0$ for $i=1,2$, then at least one of the $y_{i}$ will be greater or equal to zero. Hence $\dot{B}_{i}=S_{i}+y_{i}>0$ and $B$ is cannot be identically null, which leads to a contradiction. If $e_{i}=0$ and $e_{j}>0$, then $y_{i}=-S_{i}, y_{j}=S_{i}$ and $e_{j}=S_{i}+S_{j}$. This implies that $\dot{e_{j}}<0$. Applying similar arguments as in Theorem 3 to player $j$ leads again to a contradiction.

- Let $\phi_{1}>0$ and $\phi_{2}=0$, the interior solutions for $e_{i}$ are described below

$$
\begin{array}{ccc}
C_{1}^{\prime}\left(e_{1}\right)=\lambda_{1}, & p+\tau^{\prime}\left(y_{1}\right)+\lambda_{1}=0, \quad S_{1}-e_{1}+y_{1}=0, \quad \dot{\lambda_{1}}=\phi_{1}>0 . \\
C_{2}^{\prime}\left(e_{2}\right)=\lambda_{2}, & p+\tau^{\prime}\left(y_{2}\right)+\lambda_{2}=0, \quad \dot{B_{2}}=S_{2}-e_{2}+y_{2}, \quad \dot{\lambda_{2}}=\phi_{2}=0 .
\end{array}
$$


From these equations we obtain, on the one hand,

$$
\lambda_{1}+\lambda_{2}=-2 p, \quad \text { and then } \quad \dot{p}=-\dot{\lambda_{1}} / 2
$$

and, on the other hand

$$
\dot{p}+\dot{\lambda_{1}}+\dot{y_{1}} \tau^{\prime \prime}\left(y_{1}\right)=0 .
$$

Equations (16) and (17) imply that $\dot{\lambda_{1}} / 2+\dot{y_{1}} \tau^{\prime \prime}\left(y_{1}\right)=0$, which is a contradiction because $\dot{\lambda_{1}} / 2>0, \tau^{\prime \prime}\left(y_{1}\right) \geq 0$ and $y_{1}=e_{1}-S_{1}, \quad C_{1}^{\prime \prime}\left(e_{1}\right) \dot{e_{1}}=\dot{\lambda_{1}}>0$, Therefore, $\dot{y}_{1}=\dot{e_{1}}-\dot{S}_{1}>0$.

Now that we proved that an equilibrium for the firms (countries) must satisfy $B(t)>0$ for $t \in(0, T)$, and $B(0)=B(T)=0$, the next step is to characterize the equilibrium solutions for each firm (country) in the two cases of zero (with) and positive (without) transaction costs. For the purpose of this comparison, we denote with $J_{i}^{* 0}$ agent $i$ 's minimal cost without transaction cost and with $J_{i}^{* \tau}$ agent $i$ 's minimal cost, still in the case $r=0$. The main result of the Theorem 8 below is that efficiency is lost when transaction costs are non-negative.

Theorem 9 Assume the discount rate to be zero. Then:

a) The interior equilibrium solutions for the two firms (countries) have to satisfy the following conditions:

$$
\frac{\bar{S}_{1}+\bar{S}_{2}}{T}=e_{1}+e_{2}, \quad C_{i}^{\prime}\left(e_{i}\right)=-p+\tau^{\prime}\left(y_{i}\right), \quad \frac{\bar{S}_{i}+\bar{y}_{i}}{T}=e_{i}, i=1,2
$$

where $\bar{S}_{i}=\int_{0}^{T} S_{i}(t) d t$.

b) When there is no transaction cost paid by the firms (countries) and optimal emissions are such that $e_{i}^{* *}>0$,

$$
e_{i}^{* *}=e_{i}^{*}, \quad J^{* *}=J_{1}^{* 0}+J_{2}^{* 0},
$$

$y_{i}$ and $p$ can be non-constant functions of time and there may exist an infinite number of solutions. The equilibrium price of permits is characterized by:

$$
-p=\lambda_{1}=\lambda_{2}=\Lambda \text {. }
$$

c) In the case where the firms (countries) have to pay transaction costs, emission rates $e_{i}$, quantity of permits traded $y_{i}$, and permit's unit price $p$ are all constant.

d) The minimum total cost for the two firms (countries) under the three different scenarios compare as follow:

$$
J^{* *}=J_{1}^{* 0}+J_{2}^{* 0}<J_{1}^{* \tau}+J_{2}^{* \tau} .
$$

Proof. From Theorem 8, $\phi_{i}=0, i=1,2$. Considering interior solutions, this implies that $\lambda_{i}$ and $e_{i}$ are both constant. $B(T)=0$ implies $\frac{\bar{S}_{i}+\bar{y}_{i}}{T}=e_{i}, \quad i=1,2$. Furthermore, $y_{1}+y_{2}=0$ implies $\frac{\bar{S}_{1}+\bar{S}_{2}}{T}=e_{1}+e_{2}$, and the first-order condition (13) implies $C_{i}^{\prime}\left(e_{i}\right)=-p+\tau^{\prime}\left(y_{i}\right) i=1,2$. Note that as $\tau(y)=\tau(-y)$, we have $C_{1}^{\prime}\left(e_{1}\right)+C_{2}^{\prime}\left(e_{2}\right)=-2 p$. So $p$ is constant and hence $y_{i}(i=1,2)$ are constant as well.

When $\tau(y)$ is identically null, $C_{1}^{\prime}\left(e_{1}\right)=C_{2}^{\prime}\left(e_{2}\right)$. Henceforth, $e_{i}^{* *}=e_{i}^{*}$ and trivially $J^{* *}=J_{1}^{*}+J_{2}^{*}$. Note that in this case there exist many possible solutions for $y_{i}$ that lead to the following equilibria

$$
\dot{B}_{i}=S_{i}-e_{i}+y_{i}, \quad B_{i}(0)=0, \quad \frac{\bar{S}_{i}+\bar{y}_{i}}{T}=e_{i}, \quad B_{i}(t)>0, t \in(0, T), i=1,2 .
$$

Finally to prove item (d) of Theorem 8 , we conduct the following comparisons using the different outcomes found under the different scenarios with and without discount rate and involving positive and null transaction costs:

Using zero discount rate, both in the case with and without transaction cost, at equilibrium we have $\frac{\overline{S_{1}}+\overline{S_{2}}}{T}=e_{1}+e_{2}$, and the interior solutions for the firm's (country's) problem satisfy $C_{1}^{\prime}\left(e_{1}\right)+C_{2}^{\prime}\left(e_{2}\right)=-2 p$. Furthermore, in the case where the firms (countries) do not pay a transaction cost, we know that $C_{1}^{\prime}\left(e_{1}\right)=C_{2}^{\prime}\left(e_{2}\right)=-p$. Therefore, we analyze the value of

$$
C_{1}\left(e_{1}\right)+C_{2}\left(e_{2}\right), \quad \text { such that } \frac{\bar{S}_{1}+\bar{S}_{2}}{T}=e_{1}+e_{2} .
$$

Let's define $f\left(e_{1}\right)=C_{1}\left(e_{1}\right)+C_{2}\left(\frac{\overline{S_{1}}+\overline{S_{2}}}{T}-e_{1}\right)$. The first derivative $f^{\prime}$ evaluated at an interior optimal solution gives $f^{\prime}\left(e_{1}^{*}\right)=$ $2\left(C_{1}^{\prime}\left(e_{1}^{*}\right)+p\right)$. Note that $f^{\prime \prime}>0$. To avoid confusion we call $e_{i}^{* 0}$ the optimal emission rate for the firm's (country's) 
problem without transaction cost and $e_{i}^{* t}$ the firm's (country's) optimal emission rate when paying a transaction cost. Note that $f^{\prime}\left(e_{1}^{* 0}\right)=0 . f$ being convex, we can prove that

$$
C_{1}\left(e_{1}^{* t}\right)+C_{2}\left(e_{2}^{* t}\right)>C_{1}\left(e_{1}^{* 0}\right)+C_{2}\left(e_{2}^{* 0}\right) .
$$

Following the same notations to differentiate the case with and without transaction cost we were able to prove that $J^{* *}=$ $J_{1}^{* 0}+J_{2}^{* 0}<J_{1}^{* \tau}+J_{2}^{* \tau}$.

Now, to distinguish the buyer from the seller among the two firms (countries) we use the result of Theorem 9 in the case of positive transaction cost:

$$
\frac{\bar{S}_{1}}{T}+y_{1}=e_{1}, \text { and } \quad \frac{\bar{S}_{2}}{T}-y_{1}=e_{2} .
$$

Considering the case of symmetric costs $C_{1}=C_{2}=C$, from equation $C^{\prime}\left(e_{1}\right)+C^{\prime}\left(e_{2}\right)=-2 p$, we obtain $e_{1}<e_{2}$ and from equation (19) we obtain $2 y_{1}<\frac{\overline{S_{1}}+\overline{S_{2}}}{T}$. Following the same logic, we can prove the following result that completes Theorem 9:

Theorem 10 If the firms (countries) have the same emission reduction cost functions $C_{1}=C_{2}=C$, then when transaction costs are positive, $\bar{S}_{i} \geq \bar{S}_{j}$ implies $y_{i} \leq 0$. When transaction costs are identically null, $\bar{S}_{i} \geq \bar{S}_{j}$ implies $\bar{y}_{i} \leq 0$.

The above theorem proves that the initial allocation of emission standards could determine which agent (firm or country) would be the seller and which one the buyer, more particularly in the case of symmetric cost functions the buyer would be the agent (firm or country) with the highest emission standards at the start of the planning horizon.

\section{Conclusion}

Our results confirm that without transaction costs the agents' equilibria achieve efficiency (that would be attained by a social planner or international regulator) and reach the cost as in joint cost minimization. In presence of transaction costs, the emission abatement costs would increase for trading agents, leading to loosing the efficiency results. This concurs with Stavins' (1995) and generalizes his findings to a dynamic intertemporal framework.

Our findings also indicate that emission rates at the equilibrium are efficient in the case of zero transaction costs (same as joint problem) and constant when transaction costs are non-null. Additionally, the initial allocation of emission standards can affect the direction of trades by determining which agent would be a seller or a buyer on the emission trading market.

We further prove that no banking can never be an optimal solution for one agent (firm or country) when the discount rate is null -whether transaction costs are positive or nonexistent- neither can it be an equilibrium solution in the joint minimization problem. This result is very interesting and would be important to verify whether it would hold under different conditions; such as the case $(i)$ where only one agent (either the buyer or seller) pays the transaction costs, $(i i)$ the case of a functional form for the transaction cost different from the conventional concave transaction cost, and (iii) the case where we introduce a positive cost related to banking emission permits. Each one of those cases represent an interesting venue of future research, in addition to a more general setting with more than 2 firms or countries.

\section{REFERENCES}

[1] Baumol W. J. and W.E. Oates, "The Theory of Environmental Policy", 2nd Ed., Cambridge University Press, Cambridge, UK, 1988.

[2] Cronshaw M.B. and J.B. Kruse, 1996, "Regulated Firms in Pollution Permit Markets with Banking”, Journal of Regulatory Economics, vol.9, 179-189.

[3] Cropper M.and W. Oates, 1992, ”Environmental Economics:A Survey”, Journal of Economic Literature, vol.30, 675-740.

[4] Croker T.D., "The Structuring of Atmospheric Pollution Control Systems", in The Economics of Air Pollution, Harold Wolozin ed., Norton, New York, 1966.

[5] Dales J.H., 1968, "Pollution, Property and Prices", University of Toronto Press, Toronto, Canada.

[6] Hahn R.W. and G.L. Hester,1989, Marketable Prmits: Lessons for Theory and Practice”, Ecology Law Quarterly, vol.16, 316-406.

[7] Montgomery W.D. ,1972, "Markets in Licence and Efficient Pollution Control programs", Journal of Economic Theory, vol. 5, 395-418. 
[8] Rubin J. D., 1996, "A Model of Intertemporal Emission Trading, Banking and Borrowing”, Journal of Environmental Economics and Management, vol. 31, pp. 269-286.

[9] Stavins R. N., 1995, "Transaction Costs and Tradable Permits", Journal of Environmental Economics and Management, vol. 29 , pp. 133-148.

[10] Tientenberg T., 1985, "Emission Trading, an exercise in Reforming Pollution Policy", Resources for the Future, Washington, D.C.

[11] Tripp and D.J. Dudek, 1989, ’Institutional Guidelines for Deseigning Successful Transferable Rights Programs, Yale Journal on Regulation, vol.6, 369-391. 